Original article

Paediatrics Today 2016;12(1):160-168

DOI $10.5457 / \mathrm{p} 2005-114.152$

\title{
PHYSICAL ACTIVITY AND HEALTH-PROMOTING LIFESTYLE OF FIRST AND SECOND YEAR MEDICAL STUDENTS
}

\author{
${ }^{1}$ University of Zagreb, School of Medicine \\ Physical Education Department, Zagreb \\ Croatia, "4You" Sport and Recreation \\ Association, Zagreb, Croatia, ${ }^{3}$ University \\ of Zagreb, School of Medicine, Andrija \\ Stampar School of Public Health \\ Department of Social Medicine and \\ Organisation of Health Care, Zagreb \\ Croatia
}

Tonći MAŠINA ${ }^{1}$, Višnja KRALJIĆ ${ }^{2}$, Vera MUSIL ${ }^{3}$

\author{
Correspondence: \\ tonci.masina@mef.hr \\ Tel.: + 385981755332 \\ Fax.: + 38514684441
}

Received: June 20, 2016

Accepted: August 5, 2016

Key words: Physical activity Lifestyle - Student health.

\begin{abstract}
Objective - The aim of the study was to examine the relationship between physical activity intensity levels and health-promoting lifestyle among Croatian first and second year medical students. Materials and methods - The cross sectional study was conducted on a sample of first and second year students $(n=885)$. Data were collected by means of a self-administered anonymous questionnaire: The International Physical Activity Questionnaire (IPAQ - long form) and The HealthPromoting Lifestyle Profile [HPLP II] (Adult Version). Data were analysed by descriptive statistics and partial correlation analysis. Results - Data analysis comprised 683 participants (63\% female; response rate: $77 \%$ ). The results revealed a total physical activity score of 2661 MET-min/week and a total median HPLP II score of 2.67. The total physical activity score $(\mathrm{r}=0.190)$, total walking $(\mathrm{r}=0.187)$ and total vigorous-intensity $(\mathrm{r}=0.241)$ sub scores were significantly positive correlated with the total HPLP II; total physical activity score ( $r=0.179$; $\mathrm{r}=0.362)$, total walking $(\mathrm{r}=0.167 ; \mathrm{r}=0.210)$ and total vigorous-intensity sub scores $(r=0.177 ; r=0.5)$ with stress management and physical exercise, while total walking was correlated with health responsibility $(\mathrm{r}=0.133)$ and spiritual growth $(\mathrm{r}=0.187)$. A significant negative correlation was observed between total sitting and physical exercise $(r=-$ $0.172)$, and stress management $(r=-0.136)$. Conclusion - The results revealed a moderate level of physical activity and health-promoting life style. A low intensity level of physical activity was correlated with the health-promoting life style subscales - health responsibility and spiritual growth. The results could help physical education experts and health care providers to design health-promoting programs that support healthy options among students.
\end{abstract}

\section{Introduction}

Healthy lifestyle is closely linked with wellness and well-being. Individuals focused on incorporating the emotional, spiritual, intellectual, physical, financial, social, occupational and environmental aspects in their life are considered to have a healthy lifestyle $(1,2)$. Health risk behaviours have been connected with increased morbidity and mortality from non-communicable chronic diseases in countries worldwide $(3,4)$. Unhealthy lifestyle habits formed in childhood are transferred through adolescence to adulthood (5). Adopting healthy lifestyle habits during childhood and adolescence is important because changing habits in adulthood is difficult and demanding (6).

The main strategy for improvement of health and prevention of chronic non-com- 
municable diseases is lifestyle transformation through health-promoting activities, and should be directed towards strategies to preserve the health of individuals and communities (7). Students belong to the young adult population and they are in special focus in terms of lifestyle interventions because universities have been recognized as and are considered to be suitable settings for health promotion (8).

University students are exposed to academic stress and spend a great deal of time adapting to new circumstances (9). Student success is frequently assessed through academic achievement, which requires long periods studying in a sitting position, burdened with study obligations (10). Physical activity (PA) is considered as one of the main components of a healthy lifestyle (11). PA is defined as bodily movement produced by the skeletal muscles, which requires energy expenditure. Total energy expenditure depends on the intensity of the basal metabolic rate $(60 \%-75 \%)$, energy spent performing various forms of PA (15\%$30 \%)$ and energy for regulation of nutrition processes (about 10\%). Of the components of total energy consumption, it is possible to influence the energy required to perform various forms of PA. In extremely active persons, this can be increased by up to $50 \%(11,12)$.

The health, psychological, social, economic and environmental benefits of PA have been well documented in many studies (13-15). Therefore, guidelines and recommendations for PA have been developed for different age groups. Adults aged 18-64 years should do at least 150 minutes of aerobic moderate-intensity PA or 75 minutes of vigorous-intensity weekly (16) Children and adolescents should do a minimum of $60 \mathrm{mi}-$ nutes of medium or high-intensity PA daily (17). A commonly used measurement for PA is intensity level, determined by frequency, intensity, duration and various other indices. PA intensity level may be categorised as low, moderate or high (18). PA in adults aged 18 64 years includes leisure time, transportation (walking or cycling), work, household and yard chores, sports or planned exercise in the context of daily family and community activities $(16,18)$. Physical exercise is a part of PA that is planned, structured and repetitive, and has as the final goal the improvement or maintenance of physical fitness. Physical fitness is defined as a set of elements that are either health- or skill-related (12). Many studies have shown that new circumstances related to university education significantly impact lifestyle habits and PA. Although a predominantly moderate level of health-promoting lifestyle and PA have been observed, a low level of health responsibility, physical exercise and long periods sitting have raised concern (19-25).

The aim of this study was to examine the relationship between PA intensity levels and health-promoting lifestyle among first and second year Croatian medical students.

\section{Material and methods}

\section{Design, setting, sample}

The study was conducted among a target population of first and second year students ( $\mathrm{n}=885)$ at the University of Zagreb, School of Medicine who attended physical education course and agreed to participate in the study. The study was conducted during the academic years 2013/2014 and 2014/2015, from March to May. Data were collected by means of a self-administered anonymous questionnaire during the course classes. Date on sex and age in years were collected from the participants who agreed to participate in the study. Students returned the completed questionnaires in sealed envelopes.

\section{Instruments}

Health-promoting lifestyle was assessed using The Health-Promoting Lifestyle Profile 
[HPLP II] (Adult Version) questionnaire. HPLP II is composed of 52 statements divided into six subscales. The subscales are: health responsibility, physical exercise, nutritional habits, spiritual growth, interpersonal relations and stress management. Health responsibility is about the importance of improving an individual's health and the health of others. Physical exercise includes adhering to regular exercise patterns. Nutritional habits include establishing meal patterns and making food choices. Spiritual growth includes attaining self-actualization and fulfilment. Interpersonal relations deal with maintenance of relationships involving a sense of intimacy and closeness. Stress management includes recognizing sources of stress and taking action to control stress and achieve relaxation.

The scale is of the 4-point Likert-type and there are 4 choices for each statement, scored from 1 to 4. "Very uncharacteristic of me" receives 1 point, "somewhat uncharacteristic of me" 2 points, "somewhat characteristic of me" 3 points and "very characteristic of me" 4 points. For the English version of the HPLP II a Cronbach's alpha reliability coefficient has been reported of 0.94 for the overall scale and from 0.79 to 0.87 for the six subscales (26). For the purpose of this study, the original English version of the HPLP II was translated into Croatian. Cronbach's alpha for the Croatian version of the HPLP II was 0.896 and for the subscales it ranged from 0.65 to 0.79 . For all participants a total score and individual subscale scores were calculated.

The Croatian version of The International Physical Activity Questionnaire (IPAQ - long form) with a last seven days reference period was used to assess PA intensity level (27). The IPAQ - long form consists of 27 questions that cover the domains of PA: at work, in transport, domestic and garden activities, leisure-time and time spent sitting. Energy expenditure in a specific activity category was calculated by multiplying the weekly frequ- ency, usual duration and metabolic equivalent of the respective activity category, and was presented as metabolic equivalent-minutes per week (MET min/week). Total PA score and domain sub scores were calculated: total walking, total moderate-intensity, and total vigorous-intensity PA. Total walking was determined by walking at work, in transport and in leisure time. Total moderate-intensity sub score was determined by moderate intensity of PA at work, in the yard and household chores and leisure time, by cycling for transportation and by vigorous intensity of yard chores. Total vigorous-intensity sub score was determined by the vigorous intensity of PA at work and in leisure time. Total PA score was determined by the sum of total walking, total moderate-intensity and total vigorousintensity sub scores. Total sitting expressed the sum of minutes spent in a sitting position over seven days (min/week) $(18,27)$.

\section{Ethical considerations}

Students were approached during a course class and invited to participate in the study. They were informed of the purpose of the study and were told that participation was voluntary. They had the right to withdraw their participation at any time without any adverse impact on their studies. The written informed consent of the students was obtained prior to data collection. The study protocol was approved by the University of Zagreb, School of Medicine administration, and permission was obtained from the Ethics Committee (number: 380-59-10106-1620/159). The study was performed in accordance with the Helsinki Declaration.

\section{Statistical analysis}

Distribution of variables was tested for normality by the Kolmogorov-Smirnov test. Data were analysed by descriptive statistics and non-parametrical tests. Partial correla- 
tion coefficients controlled to gender and study year were calculated to examine associations between PA intensity levels (IPAQ - long form) and health-promoting lifestyle characteristics (HPLP II). P values less than 0.05 were considered as statistically significant. Data were entered and analysed using STATISTICA version 10.0 (Stat Soft. Inc. Tulsa, US, 2010).

\section{Results}

Analysis included data from 683 (63\% girls) students who agreed to participate in the study, and completed and returned both questionnaires, giving a response rate of $77 \%$. Group 1, in the second year of study in academic year 2013/2014, comprised 192 students (60\% female). Group 2 , in the second year of study in academic year 2014/2015, 237 students (65\% female) and Group 3 in the first year of study in academic year 2014/2015, 254 students (64\% female). Response rates in Groups 1, 2 and 3 were $64 \%, 82 \%$ and $85 \%$, respectively. Due to the perceived non-parametric distribution of the HPLP II results, the median (M) was taken as a measure of mean value and percentile as a measure of dispersion. According to Peker et al. interpretation of results range in percentiles (25-75) was as follows: from 1.60 to 2.25 represents low level, from 2.26 to 2.71 medium level and from 2.72 to 3.27 high level (28). The median score of the total HPLP II showed medium level $(\mathrm{M}=2.67$; $\mathrm{IQR}=2.44-2.90)$. A high median score was revealed in the subscales: interpersonal relations $(\mathrm{M}=3.22$; $\mathrm{IQR}=3.00-3.56)$, nutritional habits $(\mathrm{M}=2.78$; $\mathrm{IQR}=2.56-3.11)$ and spiritual growth $(\mathrm{M}=3.00 ; \mathrm{IQR}=2.67-3.33)$. A medium median score was revealed in the subscales physical exercise $(\mathrm{M}=2.38 ; \mathrm{IQR}=2.00-2.88)$ and stress management $(\mathrm{M}=2.38$; $\mathrm{IQR}=2.13$ 2.75). A low median score was revealed in the subscale health responsibility $(\mathrm{M}=2.11$; $\mathrm{IQR}=1.89-2.44$ ) (Table 1).

Analysis included the complete data from the returned IPAQ - long form questionnaires. All 683 students that agreed to participate in the study completed and returned the questionnaires, but some questions in the IPAQ - long form remained unanswered or were incomplete, therefore these were excluded from further analysis. The median of total PA score was 2661 MET-min/week. The total walking-intensity sub score was $1188 \mathrm{MET}-\mathrm{min} / \mathrm{week}$, the total moderateintensity sub score $540 \mathrm{MET}$-min/week, and the total vigorous intensity sub score 1440 MET-min/week. Total sitting was $2940 \mathrm{~min} /$ week (Table 2).

A significant positive correlation was revealed in: total PA score $(r=0.190, p<0.001)$, total walking $(\mathrm{r}=0.187, \mathrm{p}<0.001)$, total vigorous $(\mathrm{r}=0.241, \mathrm{p}<0.001)$ and total HPLP II; total PA score $(r=0.179, \mathrm{p}<0.001)$, total

Table 1 Descriptive statistics of Health-Promoting Lifestyle Profile II (HPLP II)

\begin{tabular}{lllllllll}
$\begin{array}{l}\text { HPLP II } \\
\text { subscales and total }\end{array}$ & $\mathrm{n}$ & Mean & SD & Min & Max & \multicolumn{3}{c}{ Percentile (Median) } \\
\cline { 7 - 10 } & & & & & & & & \\
Interpersonal relations & 683 & 3.21 & 0.40 & 1.78 & 4.00 & 3.00 & 3.22 & 3.56 \\
Nutritional habits & 683 & 2.78 & 0.43 & 1.56 & 4.00 & 2.56 & 2.78 & 3.11 \\
Health responsibility & 683 & 2.18 & 0.50 & 1.00 & 3.89 & 1.89 & 2.11 & 2.44 \\
Physical exercise & 683 & 2.42 & 0.61 & 1.00 & 4.00 & 2.00 & 2.38 & 2.88 \\
Stress management & 683 & 2.40 & 0.44 & 1.00 & 4.00 & 2.13 & 2.38 & 2.75 \\
Spiritual growth & 683 & 2.99 & 0.48 & 1.44 & 4.00 & 2.67 & 3.00 & 3.33 \\
HPLP II total & 683 & 2.67 & 0.33 & 1.69 & 3.88 & 2.44 & 2.67 & 2.90 \\
\hline
\end{tabular}


Table 2 Descriptive statistics of The International Physical Activity Questionnaire (IPAQ - long form)

\begin{tabular}{lllll}
\hline \multirow{2}{*}{$\begin{array}{l}\text { The scores of IPAQ - long form } \\
\text { (MET-min/week) }\end{array}$} & $\mathrm{n}$ & \multicolumn{3}{l}{ Percentile (Median) } \\
\cline { 5 - 5 } & & 25. & 50. & 75. \\
\hline Total physical activity score & 674 & 1456.00 & 2661.00 & 4385.75 \\
Total walking sub score & 660 & 713.63 & 1188.00 & 2079.00 \\
Total moderate-intensity sub score & 552 & 270.00 & 540.00 & 1200.00 \\
Total vigorous-intensity sub score & 334 & 720.00 & 1440.00 & 2880.00 \\
Total sitting (min/week) & 679 & 2160.00 & 2940.00 & 3720.00 \\
\hline
\end{tabular}

\section{Table 3 Partial correlation between physical activity intensity levels and health-promoting life-style}

\begin{tabular}{llllllll}
\hline $\begin{array}{l}\text { Partial correlation (r) } \\
\text { between IPAQ - long } \\
\text { form and HPLP II }\end{array}$ & $\begin{array}{l}\text { Interpersonal } \\
\text { relations }\end{array}$ & $\begin{array}{l}\text { Nutritional } \\
\text { habits }\end{array}$ & $\begin{array}{l}\text { Health } \\
\text { responsibility }\end{array}$ & $\begin{array}{l}\text { Physical } \\
\text { exercise }\end{array}$ & $\begin{array}{l}\text { Stress } \\
\text { management }\end{array}$ & $\begin{array}{l}\text { Spiritual } \\
\text { growth }\end{array}$ & $\begin{array}{l}\text { HPLP II } \\
\text { total }\end{array}$ \\
\hline $\begin{array}{l}\text { Total physical activity } \\
\text { score (MET-min/week) }\end{array}$ & 0.001 & -0.030 & 0.092 & $0.362^{* *}$ & $0.179^{* *}$ & 0.095 & $0.190^{* *}$ \\
$\begin{array}{l}\text { Total walking sub score } \\
\text { (MET-min/week) }\end{array}$ & 0.068 & -0.056 & $0.133^{*}$ & $0.210^{* *}$ & $0.167^{* *}$ & $0.187^{* *}$ & $0.187^{* *}$ \\
$\begin{array}{l}\text { Total moderate- } \\
\text { intensity sub score } \\
\text { (MET-min/week) }\end{array}$ & -0.042 & -0.110 & 0.041 & 0.088 & 0.047 & -0.006 & 0.012 \\
$\begin{array}{l}\text { Total vigorous-intensity } \\
\text { sub score (MET-min/ } \\
\text { week) }\end{array}$ & 0.046 & 0.097 & -0.011 & $0.500^{* *}$ & $0.177^{* *}$ & 0.098 & $0.241^{* *}$ \\
\begin{tabular}{l} 
Total sitting (min/week) \\
\hline
\end{tabular} & 0.050 & 0.004 & -0.049 & $-0.172^{* *}$ & $-0.136^{*}$ & -0.058 & -0.098 \\
\hline
\end{tabular}

IPAQ - long form=International Physical Activity Questionnaire - long form; HPLP II=Health-Promoting Lifestyle Profile [HPLP II] (Adult Version); ${ }^{*} \mathrm{p}<0.05 ;{ }^{* *} \mathrm{p}<0.001$.

walking $(\mathrm{r}=0.167, \mathrm{p}<0.001)$, total vigorous $(\mathrm{r}=0.177, \mathrm{p}<0.001)$ and subscale stress management; total walking and health responsibility $(\mathrm{r}=0.133, \mathrm{p}<0.05)$ and spiritual growth $(\mathrm{r}=0.187, \mathrm{p}<0.001)$; total PA score $(\mathrm{r}=0.362$, $\mathrm{p}<0.001)$, total walking $(\mathrm{r}=0.210, \mathrm{p}<0.001)$ and total vigorous $(\mathrm{r}=0.500, \mathrm{p}<0.001)$ and subscale of physical exercise. Total sitting was negatively correlated with physical exercise $(\mathrm{r}=-0.172, \mathrm{p}<0.001)$ and stress management $(\mathrm{r}=-0.136 ; \mathrm{p}<0.05)($ Table 3$)$.

\section{Discussion}

The results of our study showed a moderate level of health-promoting lifestyle and PA among first and second year students at the University of Zagreb, School of Medicine. In total health promoting lifestyle, students scored at medium level, while a high score was obtained on the subscales: interpersonal relations, nutritional habits and spiritual growth. A medium score was obtained for physical exercise and stress management, and a low score for health responsibility. The correlation of PA intensity level and health promoting life style showed a link between total PA score, total walking, total vigorousintensity level and total health-promoting lifestyle and the subscales: physical exercise and stress management. Total walking, as a low level of PA, was correlated with health responsibility and spiritual growth. The total moderate-intensity level of PA was not significantly correlated with health promoting lifestyle, while a negative correlation was observed between sitting and physical exercise. 
Health-promoting lifestyle and PA among university students were commonly examined separately. Peker et al. revealed among freshman dental students at Istanbul University in Turkey, a medium overall HPLP II mean score with a high score for spiritual growth, and low for health responsibility (28). Hong et al. reported among undergraduate nursing students at Mahidol University in Turkey, a medium overall HPLP II mean score in more than half the students, with the best performance in interpersonal relations, and the worst in physical exercise (23). Wei et al. revealed among Japanese university students a medium overall HPLP II mean score with the highest score for interpersonal relations and the lowest for health responsibility (29). Some of the studies revealed a non-healthy lifestyle among university students. Ulla Diez et al. reported among freshman year students at a Psychology School in Mexico, a low level of HPLP II mean score with a coherent pattern for most subscales (30). Al-Khawaldeh reported a low score in total HPLP II among Jordanian university students. They scored moderately in the areas of spiritual growth, interpersonal relations and stress management, and low in physical exercise, nutritional habits and health responsibility (31). In the health responsibility subscale, a low score was observed in numerous studies. This might be due to the fact that students have good health at that age and do not perceive responsibility for health as something that requires extra attention $(21,23,28,29$, $31)$. In concordance with this, Wolf et al. observed among college students in United States (USA) a decline in interest for medical examination at the end of the academic year, compared to the beginning (21). The observed link between low PA intensity level and the subscales health responsibility and spiritual growth in our study could raise concern about the organization of health care for students. Recommended levels of PA have been determined in relation to leisure-time, while the domains work, transport, domestic and garden have not been equally considered. Therefore, studies using the IPAQ could find considerably lower levels of physical inactivity among the student population $(32,33)$. Results of the study conducted among a representative sample of the Croatian adult population by Jurakić et al. revealed, among participants aged 15 to 24 years, a total PA score of 42.7 MET-hour/week, mainly due to a low level of PA in the domestic and garden domains. Although they reported the highest PA score in the leisure time domain, it was apparently not enough to increase their total PA score (32). Similar results were observed in our study, where the total PA score was $2661 \mathrm{MET}-\mathrm{min} /$ week, that may be considered as equivalent to the moderate category (18) It seems that first and second year medical students often engage in either walking or vigorous PA. The low total moderate-intensity sub score could be explained by the same reason as explained by Jurakić et al. (32). Students mostly neither work (do not have a job), nor work in a yard or house. Fagaras et al. reported a total PA score of 5343.92 MET-min/week among university students of physical education and physical therapy at the University in Romania (33), which is noticeably higher compared to the results obtained in our study. The total sitting of 2940 $\mathrm{min} /$ week revealed in our study indicated the large amount of time spend in a sitting position while either studying or attending the classes. Similar results were obtained by Buckworth et al. among college students in the USA. They spent almost 30 hours weekly in a sitting position while studying, although sedentary behaviours were negatively correlated with PA (19). Compared to Croatian medical students, this is almost 20 hours less that underlines the large extent of study obligations for medical students that require sitting. A low level of PA was observed among Croatian university students in other studies. Matković et al. observed an obvious decline 
in the PA index among University of Zagreb medical students after the beginning of studies and low participation in sporting activities (34). Curković reported a low level of PA and a low level of physical exercise among students of the University of Zagreb. Only $20.2 \%$ students reached the recommended frequency and duration of PA (35). The role of physical exercise is pointed out as a mechanism not just for improvement of physical condition and health that could empower defence mechanisms against stress, and contribute to greater self-esteem and better socialization (36). According to results of our study, more physically active students exercised more regularly and had better stress management skills. It seems that Croatian medical students try to compensate for long periods of sitting while studying with regular exercise in their leisure time and therefore had a tendency to improve their life style habits.

The strength of the study is the high response rate and coverage of a sample of first and second year students with extensive information about lifestyle, PA and relation between them.

The limitations of the study are: its crosssectional nature and self-reported healthy behaviours and PA. A particular weakness could be use of the IPAQ questionnaire for the university student population. PA was considered in the domains of leisure-time, work, transport, domestic and garden. PA in the domains: work, domestic and garden is generally not the type of PA performed by students in Croatia, therefore the results in particular total sub scores are noticeably low. The results of the study could not be generalized to the entire population of university students in Croatia, because only first and second year students were recruited from one specific faculty.

A suggestion for further research is conducting a study with a representative sample of university students from different facul- ties and all years of study. In addition to data about PA and health-promoting life-style (collected by questionnaires), data about body composition (body mass index, total body fat and distribution), motor ability, aerobic capacity and musculoskeletal function (muscle strength, endurance and flexibility) should be collected. This would help in determination of anthropometric characteristics, and also be a foundation for follow-up and evaluation of programs for health-promoting life style.

\section{Conclusion}

The results of the study showed a moderate level of PA and health-promoting life style among Croatian medical students. A low intensity level of PA was correlated with the health-promoting life style subscales: health responsibility and spiritual growth. The results of the study could help physical education experts and health care providers to design and develop health-promoting programs that encourage students to choose healthy options.

Acknowledgement: We would like to thank to Associate Professor, Milan Milošević, MD, PhD for the statistical analysis. The authors are grateful to the medical students who gave their time to participate and contribute to this study.

Authors' contributions: Conception and design: TM; Acquisition, analysis and interpretation of data: TM; Drafting the article: TM; Revising the article critically for intellectual content: VK and VM; Approved final version of the manuscript: TM, VK and VM.

Conflict of interest: The authors declare that they have no conflict of interest.

\section{References}

1. World Health Organization. Regional Office for Europe [homepage on Internet]. A healthy lifestyle. [cited 2016 July 15]. Available from: http://www. euro.who.int/en/health-topics/disease-prevention/ nutrition/a-healthy-lifestyle. 
2. Centers for Disease Control and Prevention [homepage on Internet]. Health-Related Quality of Life (HRQOL) - Well-Being Concepts. [cited 2016 July 15]. Available from. http:// www.cdc. gov/hrqol/wellbeing.htm.

3. Murray CJL, Lopez AD. Measuring the global burden of disease. N Engl J Med. 2013;369:448-57.

4. Croatian Institute of Public Health [database on Internet]. Croatian Health Statistics Yearbook 2015. [cited 2016 July 16]. Available from: http:// www.hzjz.hr/wpcontent/uploads/2016/05/Ljetopis_2015.

5. Hoyt LT, Chase-Lansdale PL, McDade TW, Adam EK. Positive Youth, Healthy Adults: Does Positive Well-Being in Adolescence Predict Better Perceived Health and Fewer Risky Health Behaviours in Young Adulthood? Journal Adolesc Health. 2012;50:66-73.

6. Prochaska JJ, Prochaska JO. A Review of Multiple Health Behavior Change Interventions for Primary Prevention. Am J Lifestyle Med. 2011;5(3).

7. World Health Organization. World Health Report 2002. Reducing risks, promoting healthy life. Geneva: WHO; 2002.

8. World Health Organization, Regional Office for Europe. Health Promoting Universities (edited by Tsouros AD, Dowding G, Thompson J, Dooris M). Copenhagen: WHO Regional Office for Europe; 1998 .

9. Deasy C, Coughlan B, Pironom J, Jourdan D, Mannix-McNamara P. Psychological distress and coping amongst higher education students: a mixed method enquiry. PLoS One. 2014;9(12):e115193.

10. Tartas M, Walkiewicz M, Budziński W, Majkowicz M, Wójcikiewicz K, Zdun-Ryżewska A. The coping strategies during medical education predict style of success in medical career: a 10-year longitudinal study. BMC Med Educ. 2016;16(1):186.

11. World Health Organization [homepage on Internet]. Phisical activity 2016 Fact Sheet. [cited 2016 July 15]. Available from: http://www.who.int/mediacentre/factsheets/fs385/en/.

12. Caspersen CJ, Powell KE, Christenson GM. Physical activity, exercise, and physical fitness: Definitions and distinctions for health related research. Public Health Rep. 1985;100 (2):126-30.

13. Warburton DER, Nicol CW, Bredin SSD. Health benefits of physical activity: the evidence. CMAJ. 2006;174(6):801-9.
14. Poitras VJ, Gray CE, Borghese MM, Carson V, Chaput JP, Janssen I, et al. Systematic review of the relationships between objectively measured physical activity and health indicators in school-aged children and youth. Appl Physiol Nutr Metab. 2016;41(6 Suppl 3):S197-239.

15. C3 Collaborating for Health Review [monograph on Internet]. The benefits of physical activity on health and well-being [updated 2011 June; cited 2016 July 18]. Available from: http:// www.c3health.org/wp-content/uploads/2009/09/ C3-review-of-physical-activity-and-healthv-1-20110603.pdf.

16. World Health Organization [homepage on Internet]. Global Strategy on Diet, Physical activity and Health. Global Recommendations on PA for Health 18-64 years. [cited 2016 July 18]. Available from: http://www.who.int/dietphysicalactivity/physical-activity-recommendations-18-64 years.pdf?ua=1.

17. World Health Organization [homepage on Internet]. Global Strategy on Diet, Physical activity and Health. PA and young people. Recommended levels of PA for children aged 5 - 17 years. [cited 2016 July 18]. Available from: http://www.who.int/dietphysicalactivity/factsheet_young_people/en/.

18. Guidelines for Data Processing and Analysis of the International Physical activity Questionnaire (IPAQ) - Short and Long Forms November 2005 [homepage on Internet]. [cited 2016 May 18]. Available from: https:/www.researchgate.net.

19. Buckworth J, Nigg C. Physical activity, exercise and sedentary behaviour in college students. J Am Coll Health. 2004;53:28-34.

20. Nacar M, Baykan Z, Cetinkaya F, Arslantas D, Ozer A, Coskun O, et al. Health-promoting lifestyle behaviour in medical students: a multicentre study from Turkey. Asian Pac J Cancer Prev. 2014;15:8969-74.

21. Wolf TM, Kissling GE. Changes in Life-Style Characteristics, Health, and Mood of Freshman Medical Students. J Med Educ. 1984;59:806-14.

22. Nassar OS, Shaheen AM. Health-promoting Behaviours of University Nursing Students in Jordan. Health. 2014;6:2756-63.

23. Hong JF, Sermsri S, Keiwkamka B. Health_Promoting Lifestyles of Nursing Students in Mahidol University. J Public Health Dev. 2007;5(1):27-40.

24. Mašina T, Milošević $M$. Some determinants of behavior towards health among medical students at University of Zagreb [in Croatian]. In: Djomba JK, 
Pori M, editors. Public health aspects of physical activity. Proceedings of the 8 . Cvahte days of public health and Congress of sports recreation; 2012 October 5; Ljubljana, Slovenia. Ljubljana: School of medicine, Department of public health; 2012. p. $105-10$.

25. Geok SK, Yusof A, Lam SK, Japar S, Leong OS, Fauzee Mohd SO. Physical activity and healthpromoting lifestyle of student nurses in Malaysia. J Biosci Med. 2015;3:78-87.

26. Walker SN, Sechrist KR, Pender NJ. Health Promotion Model - Instruments to Measure Health Promoting Lifestyle: Health-Promoting Lifestyle Profile [HPLP II] 1995. (Adult Version)

27. Pedišić Ž, Jurakić D, Rakovac M, Hodak D, Dizdar D. Reliability of the Croatian long version of the International Physical Activity Questionnaire. Kinesiology. 2011;43(2):185-91.

28. Peker K, Bermek G. Predictors of Health-Promoting Behaviours among Freshman Dental Students at Istanbul University. J Dent Educ. 2011;75 (3):413-9.

29. Wei CHN, Harada K, Ueda K, Fukumoto K, Minamoto K, Ueda A. Assessment of health-promoting lifestyle profile in Japanese university students. Environ Health Prev Med. 2012;17:222-7.

30. Ulla Diez SM, Perez-Fortis A. Socio-demographic predictors of health behaviours in Mexican college students. Health Promot Int. 2009;25(1). Advance access published 29 October 2009.

31. Al-Khawaldeh OA. Health promoting lifestyles of Jordanian university students. Int J Adv Nurs Stud. 2014;3(1):27-31.

32. Jurakić D, Pedišić Z, Andrijašević M. Physical activity of Croatian population: cross-sectional study using International PA Questionnaire. CMJ. 2009;50 (2): 165-73.

33. Fagaras SP, Radub LE, Vanvuc G. The Level of Physical activity of University Students. 7th World Conference on Educational Sciences, (WCES2015), 05-07 February 2015, Novotel Athens Convention Center, Athens, Greece. Procedia - Social and Behavioral Sciences 197 (2015) 1454-7.

34. Matković A, Medić A, Meštrov M, Ivković J. Common physical activity among medical students at University of Zagreb. [in Croatian]. Hrvat. Športskomed. Vjesn. 2010;25:87-91.

35. Ćurković S. Kinesiology activity and risk behaviour of students. [dissertation in Croatian]. Zagreb: Faculty of Kinesiology, University of Zagreb; 2010.

36. Joseph RP, Royse KE, Benitez TJ, Pekmezi DW. Physical activity and quality of life among university students: exploring self-efficacy, self-esteem, and affect as potential mediators. Qual Life Res. 2014;23(2):659-67. 